\title{
DEVELOPING AN IMAGE BASED LOW-COST MOBILE MAPPING SYSTEM FOR GIS DATA ACQUISITION
}

\author{
E. Frentzos ${ }^{1}$, E. Tournas ${ }^{2}$, D. Skarlatos ${ }^{2, *}$ \\ ${ }^{1}$ Geonoesis Ltd, 7 Dositheou str, Room 102 Block A, 1071, Nicosia, Cyprus, efrentzo@geonoesis.com \\ ${ }^{2}$ Cyprus University of Technology, Civil Engineering and Geomatics Dept., \\ 2-6 Saripolou str., 3036, Limassol, Cyprus (eleftherios.tournas, dimitrios.skarlatos)@ cut.ac.cy
}

Commission I, WG I/7

KEY WORDS: Mobile Mapping Systems, Low Cost, Sensor Integration, GIS

\begin{abstract}
:
The aim of this study is to develop a low-cost mobile mapping system (MMS) with the integration of vehicle-based navigation data and stereo images acquired along vehicle paths. The system consists of a dual frequency GNSS board combined with a low-cost INS unit and two machine vision cameras that collect colour image data for road and roadside objects. The navigation data and the image acquisition are properly synchronized to associate position and attitude to each digital frame captured. In this way, upon pixel location of objects appearing on the video frames, their absolute geographical coordinates can be extracted by employing standard photogrammetric methods. Several calibration steps are implemented before survey operation: camera calibration, relative orientation between cameras and determination of rotation angles and offsets between vehicle and cameras reference frames. A software tool has been developed to facilitate and speed up the calibration procedures. Furthermore, easy object coordinate extraction is supported, either in auto mode, where the conjugate image coordinates are obtained in real time using image correlation techniques. Several surveying experiments were executed to certify and check the accuracy and efficiency of the system. From the achieved results, the developed system is efficient for collecting and positioning road spatial objects such as such as road boundaries, traffic lights, road signs, power poles, etc, more rapidly and less expensively. The obtained absolute positional accuracy is less than 1 meter, depending on the availability and quality of the GPS signal.
\end{abstract}

\section{INTRODUCTION}

\subsection{Motivation}

Road network maintenance and monitoring requires accurate spatial data and its related attributes collection in a regular basis. Collecting such kind of information is a time-consuming task and involves significant costs. In order to reduce the time and cost involved in data collection, Mobile Mapping Systems (MMS) equipped with cameras and laser scanners are frequently used. Recent developments in the area of cameras, GNSS/INS receivers, computers and algorithms for $3 \mathrm{D}$ reconstruction of the objects from images, increased the interest for low cost MMSs for spatial data collection. In this work the prototype of a low-cost MMS is presented, consisting of three subsystems: two machine vision cameras (imaging subsystem), a dual frequency GNSS receiver capable of providing RTK solutions and an INS device (positioning subsystem) and a simple notebook computer with specialized software (recording subsystem), properly synchronized to acquire georeferenced stereoscopic images.

\subsection{Short System Description}

Generally speaking, the proposed low-cost Mobile Mapping System consists of three subsystems: the imaging subsystem, that is, high-end machine vision mission cameras, the positioning subsystem consisting of low cost RTK GPS / GNSS, INS boards as well as their antennas, and finally the data recording subsystem consisting of a standard laptop together with the software developed for this purpose.
More specifically, the imaging subsystem consists of two FLIR Blackfly machine vision cameras, forming a stereo ring. Cameras are mounted on a stable plastic frame in a way that their optical axes are approximately parallel and fixed, simulating normal case in photogrammetry. The baseline of the stereo system is roughly set to $50 \mathrm{~cm}$. The cameras are connected to the notebook through a USB 3.0 port and consequent images are stored in a video format. The image acquisition is externally triggered by a PPS signal generated by the GPS receiver. In this way, the perfect simultaneity of the frames acquired by both cameras and its accurate synchronization with GPS time is guaranteed down to nanosecond accuracy. The stereo rig was used to capture a computer display showing a nanosecond timer of GPS time, and both cameras captured the display at the same time.

The positioning subsystem is composed by a low-cost GNSS receiver, an INS, and the respective antenna. GNSS receiver is also fed with RTCM data corrections during the collection process providing thus real time solutions streamed into the recording subsystem. Raw data are logged in order to provide post processing solutions afterwards, employing several open source components (e.g., RTK Lib). To improve the frequency of recording positions a GNSS-INS integrated solution that improves the frequency of recording positions from $1 \mathrm{~Hz}$ (GPS) to $100 \mathrm{~Hz}$ is used. The integrated navigation solution, provided by all integrated components, as well as post processing GNSS solutions, includes the position as well as the orientation of the vehicle (X, Y, Z, heading, roll and pitch). In our integration we assume fixed positions between GNSS receiver's samples, and

\footnotetext{
* dimitrios.skarlatos@ cut.ac.cy; phone +357 25002360; fax +357 25002806; www.photogrammetric-vision.weebly.com.
} 
interpolate intermediate positions employing the provided INS vectors in - between.

Finally, the data recording subsystem is implemented by a standard notebook with a software component explicitly developed for this purpose. This component is capable of streaming NMEA and raw data from the GNSS receiver, transmitting RTCM corrections to it, as well as, receiving navigation data from INS and images from the imaging subsystem. At the same time, it displays all gathered data (images and position on a map) to the end user, in an asynchronous manner, to help administer data collection process and ease the vehicle's navigation. Free online maps are currently used for the purpose of displaying current and past vehicle position, while the inclusion of additional vector / raster data is also feasible.

The calibration of the developed MMS is achieved by a threestep process: separate calibration of each camera (interior orientation), calibration of the stereo ring (relative orientation) and estimation of the relation between the stereo ring reference frame and the GPS-INS integrated solution (exterior orientation). Despite the added complication, the independent interior and relative calibration was preferred to a combined single step relative orientation, for robustness. Interior and relative orientation is performed in lab conditions by taking pictures of a regular grid from different angles, employing OpenCV library, while exterior orientation is provided by an initial resection step in accordance with the positioning subsystem. When the system is calibrated, it is possible to compute the coordinates of a point that is visible on both images in the geographic reference system, as well as points that are visible in consecutive images with appropriate geometry.

Several software components are included in our prototype. Apart from the data gathering software displaying real time collected data, calibration tools as well as, data processing software that is used to perform measurements and save the user collected data are developed. These software components are mainly based in open source libraries such as OpenCV for camera calibration and photogrammetric processing and FFmpeg for video handling. Moreover, a data attribute editor is planned to be integrated to facilitate GIS data extraction in the form of vector data with attributes and georeferenced images.

\subsection{Paper Structure}

The paper is divided into the following sections: We initially discus the background for the study including a detailed description of existing methods for data collection and the respective existing literature. We then proceed describing the methodology involved which includes the task of building the system, different components and their communication networks, co-registering multi-sensor data, as well as basic processing of the data and multi-sensor data integration. We continue by providing results regarding the experiments conducted and the achieved solutions and discuss the proposed framework. Finally, we close the paper with concluding remarks and future - ongoing developments regarding the proposed system, which include the employment of image recognition algorithms for traffic sign recognition and sophisticated route planning algorithms that enhance the data collection process

\section{BACKGROUND}

Over the past two decades several MMS have been developed by researchers; nowadays, high-end commercial systems already exist, developed by well-known companies in the field (Riegl, Applanix, Topcon, etc.). MMS are used to extract detailed 3D data at high resolution and accuracy in several fields, such as urban planning, 3D building modelling, virtual heritage, augmented reality, transportation, and forestry (Byungyun Y., 2019). In the area of road network monitoring, modern MMS provide high quality road-related information with considerable speed and improved object modelling. However, these systems are quite expensive, and their installation and field operation are usually complicated. In recent years, with the progress of electronics and informatics, cheaper and easier to operate systems have been proposed.

The first operational MMS were developed in the 1990s by the Center of Mapping at the Ohio State University to automate and improve the efficiency of data collection for digital mapping (Novak K., 1995). This group used a vehicle-equipped with a global positioning system (GPS), CCD cameras, and several dead reckoning sensors. In the 2000 s, to meet the increasing demand for high quality 3D data, a combination of GPS, INS/IMU, LiDAR and other dead reckoning sensors were used for positioning and data capture (Gordon P., 2010). Further, precise positioning using GPS in kinematic mode led to the possibility of direct geo-referencing of the video frames. The combination of direct geo-referencing concepts and digital image technology led to lower costs, better accuracy, and increased flexibility of MMS (Klaus, P., et all., 2012). However, the overall cost remains high due to the high precision INS and LiDAR units used and to the dedicated software tools that are necessary for multi-sensor data integration. In 2007, Google Maps Street View, generated using a vehicle-based survey, to provide street-level images supplementary to Google's maps (Anguelov, D., et all., 2010).

Considering the diversity of products that can be generated with an MMS and their value for transportation and urban planning, several attempts have been made worldwide to provide MMS solutions at a lower cost. These MMS are usually based on video cameras and low-cost single frequency GPS boards available by U-Blox since 2009. In 2012, the University of Porto presented a very low cost terrestrial mobile system, with direct-georeferencing (Madeira, S., et all., 2012). It incorporates a single frequency GPS receiver board, a low-cost gyroscope and connection to a car odometer for forward/reverse indication. The data from all sensors is constantly integrated by an internal enhanced Kalman filter, to calculate WGS84 positions once per second. However, the positional accuracy is limited by single frequency GPS and the low-resolution CCD cameras.

In 2019, U-Blox released a new high-performance dual frequency GNSS chip on the market, able to provide centimetre level accuracy at $150 €(\mathrm{U}-\mathrm{Blox}, 2020)$ while the respective boards / development kits on the market based on this chip do not exceed $300 €$. This is a significant improvement that is incorporated in the developed system, in order to provide high positional accuracy without using expensive GPS equipment.

\section{IMPLEMENTATION}

As previously mentioned, the main components of the developed system are an imaging system with two high resolution cameras for stereo image capture, a GPS/ INS navigation system for the direct georeferencing of the obtained images and a data logging subsystem for data storage and processing. The details of the selected components and the respective implementation are given in the following sections. 


\subsection{Imaging Subsystem}

The imaging subsystem of our MMS consists of two Blackfly $S$ USB3 cameras available by FLIR (https://www.flir.com). The Blackfly ${ }^{\circledR} \mathrm{S}$ leverages the industry's most advanced sensors in an ice-cube form factor. It is packed with powerful features that enable the production of high-resolution images and facilitate the application development. This includes both automatic and precise manual control over image capture and on-camera preprocessing. The selected Blackfly $\mathrm{S}$ model has the following technical specifications:

\begin{tabular}{|l|l|}
\hline \multicolumn{1}{|l|}{} \\
\hline MODEL & $\begin{array}{l}\text { BFS-U3-50S5C-C: 5.0 MP, 35 FPS, } \\
\text { SONY IMX264, COLOR }\end{array}$ \\
\hline Frame Rate & 35 FPS \\
\hline Lens Mount & C-mount \\
\hline Pixel Size & $3.45 \mu \mathrm{m}$ \\
\hline Resolution & $2448 \times 2048$ pixels \\
\hline Sensor Type & CMOS \\
\hline Sensor Format & $2 / 3 "$ \\
\hline ADC & 12 -bit \\
\hline Megapixels & 5.0 MP \\
\hline Readout Method & Global shutter \\
\hline Sensor Name & Sony IMX264 \\
\hline Interface & USB 3.1 Gen 1 \\
\hline
\end{tabular}

Table 1: Imaging System specifications

The two cameras are mounted on a fixed distance to form a stereo rig, able to capture stereoscopic views with $>=80 \%$ overlap along the vehicle track. Taking as an example a mobile mapping vehicle that is being driven in an urban area at $80 \mathrm{kph}$, and the camera's frame rate, stereoscopic views at $0.64 \mathrm{~m}$ interval can be effectively captured using this equipment.

The acquisition of synchronized images, i.e. images acquired "at the same time" from both cameras, is of paramount importance. Otherwise significant positional errors will be encountered in the coordinate measurements. These errors are relatively increasing to the vehicle velocity and should be minimized as possible. In our definition "same time" is that the cameras must take start exposing each image within microseconds of each other. One approach to accomplish such a synchronized acquisition, is to use the left camera of the ring as "primary" to trigger the right camera when an image acquisition is requested by the controlling software: the two cameras are physically connected with a custom GPIO cable that sends out a strobe signal from the primary to the secondary camera to acquire images at the same time. On the other hand while this approach is effective considering lab conditions and independent stereo ring data acquisition, it should be revised when integrated with the positioning subsystem, following the methodology provided in section 3.4.

\subsection{Positioning Subsystem}

Recent advances in the field of GNSS receivers / boards, directed us towards the employment of a low cost RTK board: while commercial GNSS / GPS receivers still cost several thousand of euro, recently available board in the market are in the order of hundreds of euro, making it therefore easy to integrate them into our system. Thus, we have employed U-blox F9P board integrated into a Sparkfun's GPS-RTK2 board. This is an affordable solution, since the board's cost does not excess $300 €$. We also used a Tallysman antenna capable of receiving L1/L2 signals from GPS, GLONASS and Galileo L5; other alternatives at even lower costs currently exist in the market, e.g., (U-blox,2020). The main advantage of GPS-RTK2 board is that it can accept RTCM messages and thus achieve fixed kinematic solutions with accuracy of $0.01 \mathrm{~m}+1 \mathrm{ppm}$ CEP horizontally and $0.01 \mathrm{~m}+1 \mathrm{ppm}$ CEP vertically, and also a typical convergence time in the RTK mode of less than $10 \mathrm{sec}$. We also used a low-cost INS device, namely, XSENS MTI-7DK which measures rotation data with an RMS of $0.5^{\circ}$ Roll/pitch (static - dynamic), and $1.5^{\circ}$ Yaw (dynamic). Finally, the antenna signal was split using an appropriate signal splitter in order to direct the signal to both GPS and INS devices.

We have further implemented a software component that directly communicates with GPS-RTK2 board via one interface (serial port) serving as both receiver and transmitter. The developed software component performs the following tasks:

Configuring the device: The software initially configures the device to receive RTCM3 messages and to transmit the respective solutions (NMEA sentences), as well as the raw GNSS measurement and navigation data.

Receiving and parsing NMEA data: The employed board transmits GPS receiver data back to our software within the NMEA specification. Therefore, we developed a component that parses the respective sentences and transforms them into meaningful data displayed in the respective user interface.

Sending RTCM corrections: RTCM corrections are gathered from NTRIP providers (CYPOS, ATLAS, URANUS etc are possible available providers in Cyprus) and transmitted to the receiver.

We have tested our approach and found out that the time required to get a fix from the board is in the range of 20 seconds to 1 minute, while this fix is maintained under good circumstances (atmospheric conditions, baseline length, GNSS antenna, multipath conditions, satellite visibility and Geometry according to the product's datasheet).

Finally, we have developed a software component that communicates with INS MTI-7, using the provided SDK. The data gathered from INS do not follow the NMEA specification; several objects are provided by the SDK enumerated using the respective measured values from the device, position, and rotation (roll, pitch, yaw).

\subsection{System Calibration}

Calibration is the process of determining the interior, relative and exterior orientation of the imaging subsystem, as well as the compensation for the misalignment between it and the positioning subsystem (GPS/ INS). The misalignment involves both translation and rotation components between the cameras and the mapping reference system. 


\subsubsection{Camera Calibration}

Camera calibration is a necessary step in $3 \mathrm{D}$ computer vision to extract metric information from 2D images. The camera calibration process includes the estimation of several parameters:

- the internal geometry of each camera (interior orientation) -the relative positions between the two cameras (relative orientation)

Interior orientation is a part of camera calibration where the measurements relating to the camera, such as the perspective center and focal length are determined (OpenCV, 2019). It also involves finding the scaling, skew factor, and lens distortion. The interior orientation is performed in lab conditions by taking pictures of a regular grid from different angles (Figure 1). By transforming the image pixels to real world lengths, the metrics of imaging can be determined.

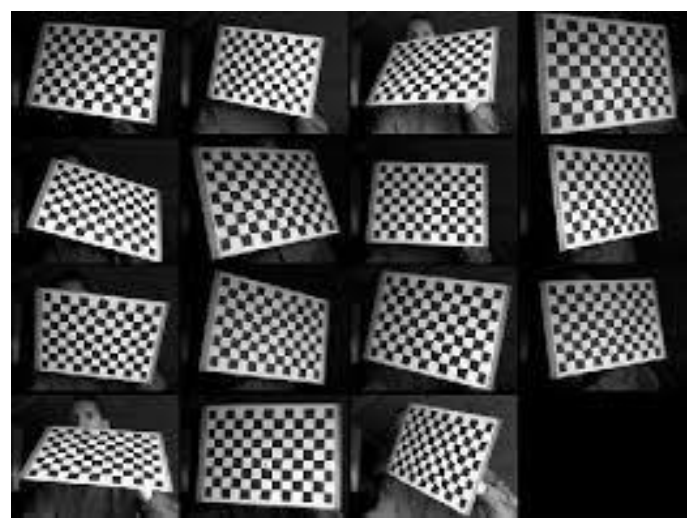

Figure 1: Chessboard calibration pattern

The camera model adopted is the "pinhole" camera modelFigure 2. In this model, a scene view is formed by projecting $3 \mathrm{D}$ points into the image plane using a perspective transformation.

$s M m^{\prime}=A[R \mid t]^{\prime}$

or

$s\left[\begin{array}{l}u \\ v \\ 1\end{array}\right]=\left[\begin{array}{ccc}f_{x} & 0 & c_{x} \\ 0 & f_{y} & c_{x} \\ 0 & 0 & 1\end{array}\right]\left[\begin{array}{lll}r_{11} & r_{12} & r_{13} t_{1} \\ r_{21} & r_{22} & r_{23} t_{2} \\ r_{31} & r_{32} & r_{33} t_{3}\end{array}\right]\left[\begin{array}{c}X \\ Y \\ Z \\ 1\end{array}\right]$

where:

$(X, Y, Z)=$ the coordinates of a $3 \mathrm{D}$ point in the world coordinate space

$(\mathrm{u}, \mathrm{v})=$ the coordinates of the projection point in pixels

$A$ is a camera matrix, or a matrix of intrinsic parameters

$\left(c_{x}, c_{y}\right)=$ the principal point that is usually at the image center

$f_{x}, f_{y}=$ the focal lengths expressed in pixel units.

Relative orientation (OpenCV, 2019) involves the estimation of transformation between two cameras making a stereo ring. In this case the relative position and orientation of two cameras is fixed, and if the rotation $\mathrm{R}$ and the translation $\mathrm{T}$ of an object relative to the first camera $(R 1, T 1)$ and to the second camera $(R 2, T 2)$ are known, then those poses definitely relate to each other. This means that, given $(R 1, T 1)$, it should be possible to compute $(R 2, T 2)$ if the position and orientation of the second camera relative to the first camera is known. That is exactly what relative orientation does. It computes $(R, T)$ so that:

$$
\begin{aligned}
& R 2=R * R 1 \\
& T 2=R * T 1+T
\end{aligned}
$$

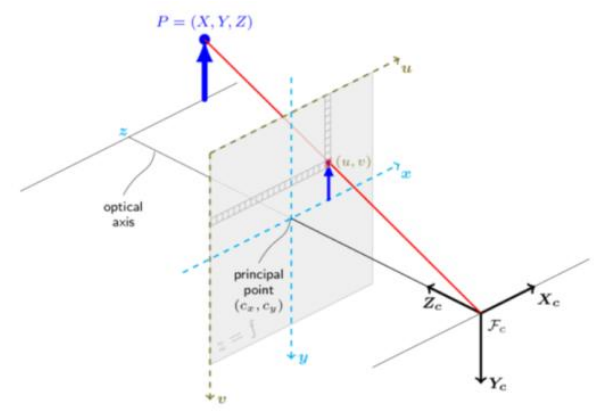

Figure 2: The pinhole camera model

The interior orientation and the relative orientation parameters are estimated in one step using the OpenCV, an open source computer vision and machine learning library (OpenCV, 2019).In order to calibrate, multiple images of a chessboard with fixed square size are used. It is recommended to have at least 30 images of the checkerboard in all possible orientations of the checkerboard to get good calibration results (Figure 1). Corner points are automatically detected on each image. These corner points in the image correspond to some 3D point in the world which that is easy to calculate, since the checkerboard has a very well-defined geometry. These point correspondences are the input data to OpenCV to estimate the calibration parameters.

\subsubsection{Boresight Misalignment}

Since the developed MMS is made up of two separate components - cameras and GPS/ INS positioning devices, these sensors record values in their separate coordinate system. Once the sensors are put together on the platform, they must be calibrated before they are deployed for data collection. Under this perspective, calibration is the compensation for the physical misalignment between different sensors belonging to the system (Mostafa, 2001). In general, the misalignment involves both translation and rotation components between the imaging system (stereo ring) and the navigation system (GPS/ INS).

In order to determine the translation and rotation values required to transform the data from the imaging reference system to a global frame, it is important to determine the relationship between physical locations of the origins of the stereo ring and the GPS/INS.

This is achieved by an initial resection (pose estimation) performed using a small control field with predefined points, collecting at the same time the INS and GPS provided measures. Specifically, the camera's exterior orientation $\operatorname{Ext}_{c}\left(R_{c}, T_{c}\right)$ is computed through pose estimation, while INS device provides its $\operatorname{Ext}_{n}\left(R_{n}, T_{n}\right)$, and RTK GPS/GNSS provides a single $T_{g}$ vector. Since the solutions $T_{g}$ gathered from GPS/GNSS device provide higher precision of the same antenna position, they are used to replace $T_{n}$ in $E x t_{n}$, computing thus the final positioning subsystem's exterior orientation $\operatorname{Ext}_{p}\left(R_{n}, T_{g}\right)$. Finally, the difference $\operatorname{Ext}_{c, p}\left(R_{c, p}, T_{c, p}\right)$ between the imaging and positioning subsystems is computed as follows: 
$R_{c, p}=R_{c} * R_{n}{ }^{T}$

$T_{c, p}=T_{c}-R_{c, p} * T_{g}$

Finally, this value of $\operatorname{Ext}_{c, p}\left(R_{c, p}, T_{c, p}\right)$ is used throughout the data collection to correct the values that are collected by the positioning subsystem, and calculate the camera's exterior orientation on each separate collected frame. Specifically, given the positioning subsystem exterior orientation $\operatorname{Ext}_{p, i}\left(R_{n, i}, T_{g, i}\right)$ at frame $i$, camera's exterior orientation $\operatorname{Ext}_{c, i}\left(R_{c . i}, T_{c . i}\right)$ is computed as follows:

$R_{c, i}=R_{c, p} * R_{n, i}$

$T_{c, i}=T_{c, p}+R_{c, p} * T_{g}$

\subsection{Synchronization}

The synchronization between the positioning and the imaging subsystems is a crucial task on MMS since small temporal difference between the imaging / positioning / recording subsystems may lead to error in the calculated positions in the order of several meters. For example, a speed of e.g., $80 \mathrm{~km} / \mathrm{h}$, translates to approximately $22.2 \mathrm{~m} / \mathrm{sec}$, and an error in the synchronization of e.g. 50 milliseconds results to $1 \mathrm{~m}$ only by this source.

On the other hand, existing GPS/GNSS boards, such as (U-blox, 2020) can be used to facilitate our problems. Specifically, such board can transmit GPS pulse-per-second signal, created by the GPS receiver in a timely manner (e.g., every second), and is used to synchronize other devices to GPS time.

Pulse per second (PPS or 1PPS) is an electrical signal with a width of less than one second and a sharply rising or abruptly falling edge that accurately repeats once per second. Precision clocks are sometimes manufactured by interfacing a PPS signal generator to processing equipment that aligns the PPS signal to the UTC second and converts it to a useful display. PPS signals have an accuracy ranging from 12 picoseconds to a few microseconds per second, or 2.0 nanoseconds to a few milliseconds per day based on the resolution and accuracy of the device generating the signal.

In our case, PPS signal transmitted by the GPS RTK2 board from its PPS interface can be captured by the imaging subsystem to be used as a trigger. Specifically, the employed cameras supply a GPIO interface that can connect them to the GPS provided PPS signal, so as to use it as a hardware trigger for taking pictures; we use this interface to connect the same PPS signal to both cameras with a single physical cable that branches to both cameras. As such the system provides synchronized image acquisition by both cameras, which is also synchronized with the PPS signal that demonstrates GPS time.

When triggered, cameras send the acquired images to the recording subsystem (laptop), with a delay which varies between $20-200$ milliseconds depending on the systems workload at the time; nevertheless such time difference shouldn't normally pose any difficulty in choosing the appropriate pairing for gathered images and received GPS signals (NMEA words). On the other hand, while NMEA words transmitted through the GPS USB interface are timestamped with GPS time, they may demonstrate grater variance in their temporal differences between the GPS timestamps and the time of the laptop at their arrival, (time of Host), mainly due to the twofold USB interface sending RTCM corrections and receiving NMEA sentences at the same time.

As such, we continuously monitor and record the time difference between Host time and GPS time. Our goal is to determine the actual signed time difference $\Delta t$ between Host time and GPS time, given the observed value $\Delta t^{\prime}$ between them, to use it to timestamp the camera acquired images. Actually, observed value $\Delta t$ ' consists of the sum of the actual $\Delta t$ which can be considered constant for a time period of several seconds, and $\delta t$ which is the delay between the time of NMEA sentence transmittal and its receival; our goal then is to minimize the value of $\delta t$ which is always positive $(\delta t \geq 0)$. Thus, we choose the minimum observed time difference $\Delta t^{\prime}$ between them during the last 100 seconds which approximates $\Delta t$. This value is continuously changing as the time evolves, allowing us to catch small $\Delta t$ deviations which may occur during a longer time period of data collection, i.e., several hours.

We have extensively tested our approach and find out that it works seamlessly providing both accurate GPS - image synchronization and correct image timestamping.

\subsection{Setup}

The developed MMS could be mounted on a variety of mobile platforms, given that the cameras, INS and GPS antenna are required to be in a stable position throughout the data collection (Figure 3). Moreover, in order to minimize electromagnetic interference to GPS signal, when setting up our frame on top of a vehicle, we acquire the initial mount's position / orientation with the cameras off, and then the GPS antenna is removed to a remote position and operates there during the data collection process. The misalignment between the collected and calibrated antenna position is calculated and added to the boresight misalignment process.

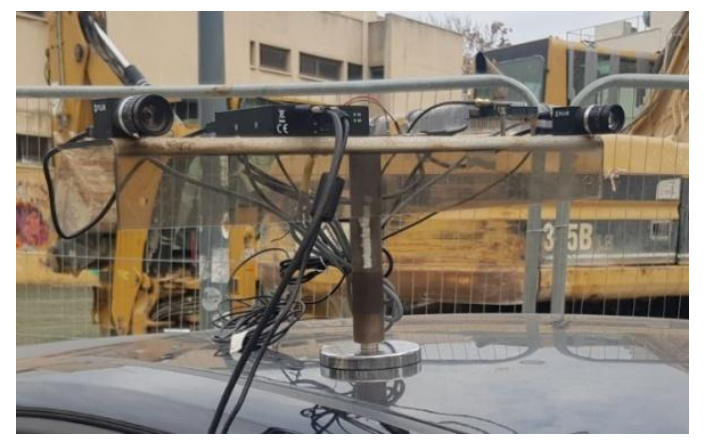

Figure 3: Mount setup

\section{SYSTEM INTEGRATION}

\subsection{Software Components}

Several software components have been developed to support our MMS. In the following sections, the calibration tool, the data collection tool and the data processing software are presented.

\subsubsection{Calibration tool}

The developed calibration tool can implement all calibration tasks, that is, interior, relative, boresight misalignment (via 
resection), and basic setup testing via photogrammetric intersection. A standard chessboard pattern is used and photographed using several positions / angles. The tool initially performs automatic chessboard corner recognition and all positions are displayed to the user, so as to be able to inspect and / or correct and / or exclude the automatically recognized points. Then, the calibration process is performed and the respective residuals (in pixels) for each observation and in total, are displayed to the user; finally, the result of the respective process is saved to the disk. A screenshot of this process is displayed in the following Figure 4 regarding the task of interior orientation, and Figure 5 regarding the relative orientation task.

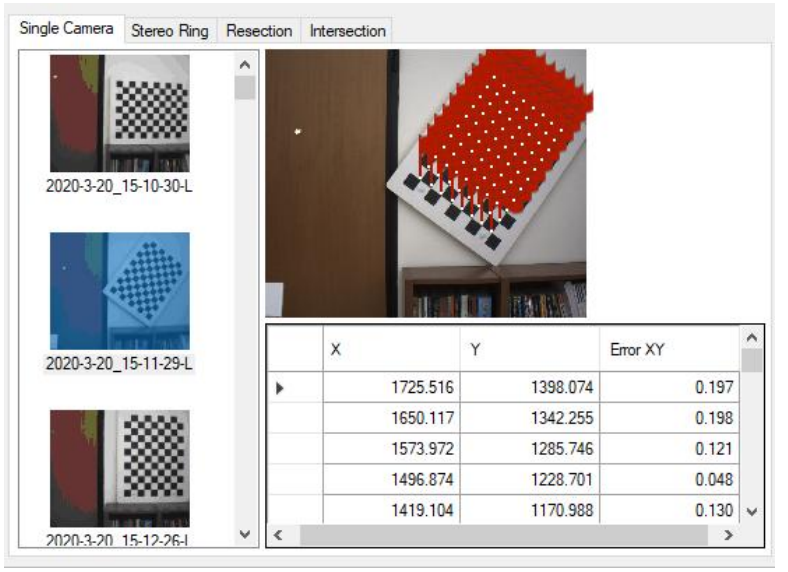

Figure 4: Example screen of single camera calibration software

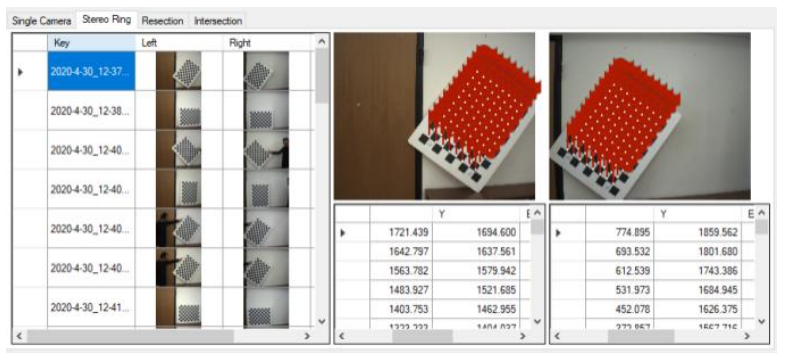

Figure 5: Example screen of stereo rig calibration software

\subsubsection{Data Collection Software}

The developed data collection software utilizes all components connected to a laptop with windows 10 and a wireless connection to the internet. The software assures the synchronization between all connected modules, that is, the GPS-RTK2 board, MTi-7 INS/GNSS and FLIR (machine vision) cameras, using the methodology described in this paper. Moreover, given the current position of the sensors, our software is capable to display the system's current position on a map, as well as all the recorded so far trajectory of the respective vehicle. The software also displays several frames of the gathered video data so as to give the user the ability to check whether all components are working on a regular manner. Summarizing, the following indications are displayed over the UI of our software

- $\quad$ GPS Condition (fix / float / autonomous / timeout)

- MTi-7 connection status (connected / timeout)

- Cameras status (gathered frames are displayed every, e.g., $5 \mathrm{sec}$ )

Regarding the map used in the background, our software currently can display raster maps, as well online XYZ tile based maps, such as google maps, Bing maps, OpenStreetMap etc. As such, the user has the ability to choose among several backgrounds which are suitable for her needs. The software also supports several projection systems for the geographically displayed data, including WGS84, UTM, Cyprus's LTM etc. On the other hand, all data collected are stored in the application's data folder in the underlying system on which data are collected, i.e., WGS84.

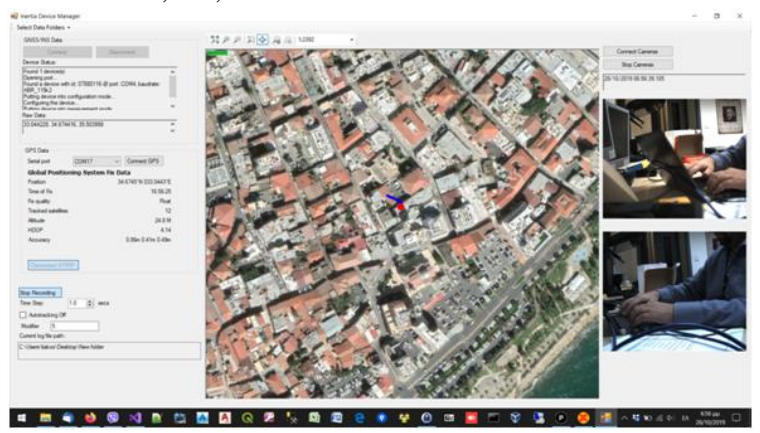

Figure 6: Example screen of data collection software

All data acquisitions are performed in an asynchronous manner, were the main UI component is regularly refreshed, while the user experiences no delays in the tasks requested, i.e., zooming - panning the map etc. An example screen of the UI that assist the video collection process is displayed in Figure 6.

We have tested the performance of the developed software on several configurations. Among the three collecting processes obviously the most demanding in terms of CPU utilization, RAM and storage utilization, is the process that captures imagery data in the form of Video files. We have currently established that a rather old i7-4510U @ 2GHz notebook, with at least three USB built in interfaces, is capable of serving the developed app and work seamlessly during a data collection procedure of several hours.

\subsubsection{Data Processing Software}

So far, we have developed a simplistic data processing software capable of performing basic data collection operations, such as 3D object position acquisition, on picture distance measuring etc, while displaying at the same time the system's current position on a map. Background maps can be selected from a variety of online maps (XYZ tiles) and other raster or vector data sources, displayed in the reference system of user preference. An example screenshot of the data processing software can be found in Figure 7.

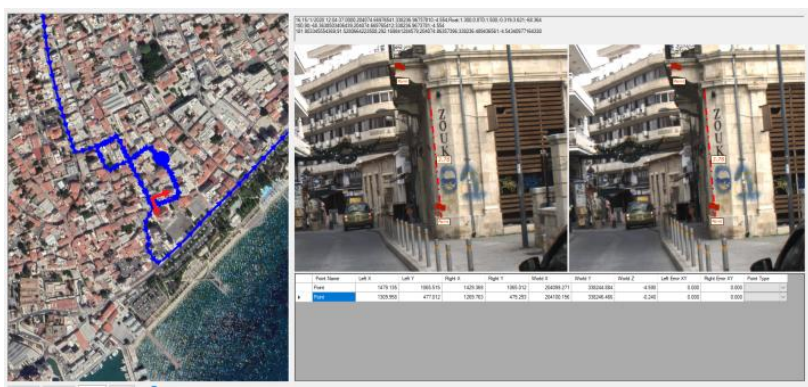

Figure 7: Example screen of data processing software

All on image operations are performed interactively monoscopically, while on the background automatic matching is performed. As such, the user may use the image of his preference (left or right) to measure points and / or dimensions. In the case the matching algorithm fails, user is informed to manually place the point in the other image and / or move the 
failed matched position to its correct place. Automatic matching is also used for zooming / panning operations inside the images in order to display the same image part at both frames simultaneously, so that user / digitizer experiences a very attractive user interface which requires only digitizing objects in a single image.

\subsection{Experiments}

To test the validity of the system, camera interiors and stereo rig relative orientation was determined used the developed software. The results of the calibration process are summarized in the following Table 1 . The respective calibration results demonstrate that accuracy is typical of the corresponding photogrammetric processes (i.e 0.5 pixels for interior and 1 pixel for relative). Therefore, we can assume that both equipment and methodologies followed are proper and results within expected limits.

\begin{tabular}{|l|c|c|c|}
\hline & $\begin{array}{c}\text { Left Camera } \\
\text { Interior }\end{array}$ & $\begin{array}{c}\text { Right Camera } \\
\text { Interior }\end{array}$ & Relative* \\
\hline $\begin{array}{l}\text { number of } \\
\text { Images }\end{array}$ & 95 & 103 & 47 \\
\hline $\begin{array}{l}\text { number of } \\
\text { observations }\end{array}$ & 8360 & 9060 & 4136 \\
\hline $\begin{array}{l}\text { Residuals } \\
\text { (pixel) }\end{array}$ & 0.504 & 0.524 & 0.94 \\
\hline
\end{tabular}

*pair of images

Table 1: Interior and relative orientation results.

Moreover, two elongated georeferenced check fields with physical targets have been measured using RTK GPS/GNSS inside two city canyons, with a length of several hundreds of meters, and a total of 40 check points. The system was initialized on top of the car and then performed at least two passes in each one of the control fields following the same direction. The collected data where then processed by a user and the predefined points where recognized and digitized on the collected data software, to compare their computed coordinates with the ones of the check field. All digitized points were at an effective distance from the cameras of no more than $20 \mathrm{~m}$; this is a reasonable assumption since consecutive pairs of frames are not expected to be separated more than $22 \mathrm{~m}$, even at working speed of $80 \mathrm{kph}$ and a frame rate of $1 \mathrm{sec}$. The results of the comparison between check points and calculated coordinates are summarized in the following Table 2.

\begin{tabular}{|l|l|l|l|}
\hline & $\mathrm{XY}(\mathrm{m})$ & $\mathrm{Z}(\mathrm{m})$ & $\begin{array}{l}\text { Distances } \\
(\%)\end{array}$ \\
\hline Average Error & 0.67 & 0.45 & 10.9 \\
\hline St Dev & 0.60 & 0.41 & 4.8 \\
\hline
\end{tabular}

Table 2: Error estimation

Clearly the positional error is less than $1 \mathrm{~m}$ horizontally and vertically. Moreover, the horizontal error is mainly concentrated along the vehicle movement axis (parallel to camera axis), with the maximum error along the stereo rig's $\mathrm{X}$ axis not exceeding $0.50 \mathrm{~m}$ at the worst case. This is an expected result given the fact that photogrammetry suffers of errors in depth calculation, and the relatively small baseline does not support better results.

A secondary test was performed to estimate the absolute accuracy of the proposed system. Within a given route, a loop was performed and XYZ coordinates of 10 points were recalculated. The average standard deviation on those 10 points in LTM93 was $\pm 0.36 \mathrm{~m}$ horizontally and $\pm 0.18 \mathrm{~m}$ vertically. This was expected as the error in camera axis was translated in $\mathrm{XY}$ error. At the same time, $\mathrm{Z}$ axis estimation doesn't suffer so much being parallel to camera plane.

\section{CONCLUSIONS - FUTURE WORK}

In this work a low-cost Mobile Mapping System (MMS) with hardware cost lower than $3.000 €$, is presented. Our MMS is calibrated based on existing well documented and tested open source software (OpenCV), which was integrated into our interface. Several software components have been developed to facilitate and speed up the calibration, data collection and data extraction procedures; easy object coordinate extraction is supported, either in auto mode, where the conjugate image coordinates are obtained in real time using image correlation techniques. Several surveying experiments were executed to certify and check the accuracy and efficiency of the system. From the achieved results, the developed system is efficient for collecting and positioning road spatial objects such as such as road boundaries, traffic lights, road signs, power poles, etc, more rapidly and less expensively. The obtained absolute positional accuracy is in less than 1 meter, depending on the availability and quality of the GPS signal.

We are currently working into the process of improving the collected data precisions following multiple intersections (across and along frame pairs), increasing mount's baseline stability, improving the system calibration, and, possibly replacing INS with photogrammetric techniques defining horizontal levels on several frames. Future work includes the improvement of the user interface to enhance automation, including the integration of automatic traffic sign recognition capabilities etc. We also plan to combine our MMS with an environment supporting attribute data extraction and storage in a spatial database, as well as GIS direct data extraction. Finally, in order to facilitate the data collection process, we plan to integrate into our data collection software a route network covering algorithm that can be adjusted in real time, providing the data collector with guidance over the road network that needs to be covered.

\section{ACKNOWLEDGEMENTS}

Work supported by the funded program MOBILO ENTERPRISES/0916 by Cyprus's Research Promotion Foundation, under contract ENTERPRISES/0916/0055

\section{REFERENCES}

Gordon P., 2010.An Introduction to the TechnologyMobile Mapping Systems. GEOInformatics, January/February 2010, $32-43$.

Novak K., 1995. Mobile mapping technology for GIS data collection. Photogramm. Eng. Remote Sens. 1995, 61,493-501.

Anguelov, D., Carole, D., Daniel, F., Christian, F., Stéphane, L.; Richard, L., Abhijit, O., Vincent, L.,Josh,W., 2010.

Google Street View: Capturing the World at Street Level. Computer 2010, 43, 32-38.

Byungyun Y.,2019. Developing a Mobile Mapping System for 3D GIS and Smart City Planning.

Klaus, P., Schwarz, N., El-Sheimy, N., 2012. Mobile mapping systems - State of the art and future trends.Int. Arch Photogramm. Remote Sens. Spat. Inf. Sci. 2012, 35, 759-768. 
Madeira, S., Goncalves, JA., Bastos, L., 2012. Sensor Integration in a Low Cost Land Mobile Mapping System. Sensors 12(3), 2935-2953.

U-Blox, 2020. ZED-F9P module, F9 high precision GNSSmodule, Documentation and resources. https://www.ublox.com/en/product/zed-f9p-module, accessed (1 May 2020).

Mostafa, M.M.R., 2001. Boresight calibration of integrated inertial/camera systems. InProceedings of the International Symposium on Kinematic Systems in Geodesy,Geomatics and Navigation (KIS 2001), Banff, AB, Canada, 5-8 June 2001.

OpenCV software library,2020. [Online]. Available: [1] https://opencv.org/. (1 May 2020].

FFmpeg Developers, 2016. ffmpeg tool (Version be1d324)

[Online]. Available: http://ffmpeg.org/ (9 May 2020) 